\title{
Generating Rooted Triangulations Without Repetitions ${ }^{1}$
}

\author{
D. Avis ${ }^{2}$
}

\begin{abstract}
We use the reverse search technique to give algorithms for generating all graphs on $n$ points that are 2- and 3-connected planar triangulations with $r$ points on the outer face. The triangulations are rooted, which means the outer face has a fixed labelling. The triangulations are produced without duplications in $O\left(n^{2}\right)$ time per triangulation. The algorithms use $O(n)$ space. A program for generating all 3-connected rooted triangulations based on this algorithm is available by ftp.
\end{abstract}

Key Words. Rooted triangulations, 2- and 3-Connected triangulations, Reverse search technique.

1. Introduction. Let $G=(V, E)$ be a planar graph with vertex set $V=\left\{v_{1}, \ldots, v_{n}\right\}$, and let $3 \leq r \leq n$ be an integer. $G$ is an $r$-rooted triangulation if it can be embedded in the plane such that the outer face has labels $\left\{v_{1}, \ldots, v_{r}\right\}$ in clockwise order, and all interior faces are triangles. A vertex (or edge) on the external face is called external, otherwise it is internal. All $r$-rooted triarigulations are 2-connected. It is well known that an $r$-rooted triangulation is 3-connected if and only if there does not exist an edge between two nonconsecutive vertices on the outer face. It follows that all 3-rooted triangulations are 3 -connected. Let $m$ be the number of edges in $G$. If follows from Euler's formula that $m=3 n-3-r$. We denote the degree of a vertex $v$ by $d(v)$.

The purpose of this paper is to describe how to generate all 2- or 3-connected rooted triangulations without repetitions. The procedure was programmed and has been used to settle two questions involving triangulations. Using a list of 3-rooted triangulations on nine points, Binhai Zhu verified that each triangulation has a set of two dominating edges. This means that each triangle of the triangulation contains at least one vertex which is an endpoint of one of the edges. This results in an improvement of the lower bound on the number of edge guards required to guard a polyhedral terrain given in [3]. Hurtado [8] asked the author if there exist Eulerian 3-rooted triangulations for $n \geq 11$, for $n$ odd, and $n$ not divisible by 3 . It was known that none exist for $n=4,5$, and 7 [8]. These results were verified, and it was found that for $n=6,8,9,10,11,12$, and 13 there are respectively $1,3,7,15,63,168$, and 561 Eulerian 3-rooted triangulations.

Two $r$-rooted triangulations are isomorphic if there is an edge preserving isomorphism between the vertex sets of the two triangulations that preserves the labelling of the outer face. For given $n, r$, let $f(n, r)$ be the number of nonisomorphic 2-connected triangulations, and let $g(n, r)$ be the number of nonisomorphic 3-connected triangulations. Observe that $f(n, 3)=g(n, 3), n \geq 3$. Tutte [12] found a closed formula for $g(n, r)$.

\footnotetext{
${ }^{1}$ This research was supported by N.S.E.R.C. Grant Number A3013, F.C.A.R. Grant Number EQ1678, and a bilateral exchange from J.S.P.S./N.S.E.R.C.

${ }^{2}$ School of Computer Science, McGill University, 3480 University, Montréal, Québec, Canada H3A 2 A7.

Received April 5, 1994; revised November 17, 1994. Communicated by F. P. Preparata.
} 
For $n \geq 5$,

$$
g(n, 3)=\frac{2}{(n-2) !}(3 n-6)(3 n-5) \cdots(4 n-11) .
$$

For $r \geq 4, n \geq r+2$,

$$
\begin{aligned}
g(n, r)= & \frac{3(r-1) !(r-4) !}{(3 n-6) !} \\
& \times \sum_{j=0}^{\min (n-r-1, r-3)} \frac{(4 n-r-8-j) !(r-1+j)(r-3-3 j)}{j !(j+1) !(r-3-j) !(r-1-j) !(n-r-j-1) !} .
\end{aligned}
$$

Brown [5] found a closed formula for $f(n, r)$. For $n \geq r \geq 3$,

$$
f(n, r)=\frac{2(2 r-3) !(4 n-2 r-5) !}{(r-1) !(r-3) !(n-r) !(3 n-r-3) !} .
$$

The efficient generation of unrooted triangulations has received some attention in the literature. This appears to be harder than generating all rooted triangulations, and isomorphism testing is required by current algorithms. This means that all nonisomorphic triangulations generated must be stored. Bowen and Fisk [4] describe a method of generating all triangulations of the sphere. Dillencourt [7] gives an algorithm for generating all simplicial polyhedra in three dimensions. This paper also gives methods for generating other classes of polyhedra. Using an approach dual to that given here, Deza et al. [6] give a method for generating all simple 3-polytopes by applying a variation of reverse search to Wagner's theorem (see Section 2.3). Their use of reverse search is somewhat different than that given here and isomorphism testing is required to remove duplicates. It is an open problem whether reverse search can be used to generate simple polytopes (or unrooted triangulations) without isomorphism testing.

In this paper we show how to generate all 3-connected $r$-rooted triangulations in $O\left(n^{2} g(n, r)\right)$ time and all 2-connected $r$-rooted triangulations in $O\left(n^{2} f(n, r)\right)$ time. We use the reverse search method developed by Fukuda and the author [1], [2]. A feature of the reverse search method is that no isomorphism test is required, and the triangulations need not be stored. The algorithm uses only $O(n)$ space. We begin by reviewing informally the reverse search method in the context of our application. It is assumed for the rest of this section that we wish to generate all 3-connected $r$-rooted triangulations on $n$ points, for given fixed $n$ and $r$. In the next section we give the details of the required procedures for the 3-connected case. Section 3 contains a description of the reverse search procedure, the data structure required, and an analysis of time and space complexity. Section 4 describes the modifications necessary to generate 2 connected triangulations. Finally in Section 5 we discuss some computational experience, open problems. possible improvements, and explain how a copy of the program may be obtained.

The reverse search method is a technique for generating all vertices of a graph whose edges are given implicitly by an oracle. Let $H=(T, U)$ be such a graph. In our application, each vertex in $T$ corresponds to an $r$-rooted triangulation with given fixed $n$ and $r . U$ is the edge set of adjacent vertices in $T$. In our application, two $r$-rooted triangulations are adjacent if they differ by exactly one edge. The reverse search method 
works by finding a spanning tree in the graph $H$. To do this we first fix some given triangulation with the required parameters $n$ and $r$. Call the vertex in $H$ corresponding to this triangulation the target $t^{*}$. We now define a local search procedure, $L$, that given any vertex $t$ of $H$ defines a unique adjacent vertex in $H$, with the property that repeated application of $L$ defines a path in $H$ to the target. In other words, $(t, L(t))$ is an edge in $U$ and $L^{k}(t)=t^{*}$ for some finite integer $k$. The path generated consists of a sequence of $r$ rooted triangulations that differ in exactly one edge and ends with the target triangulation. The set of all such paths clearly forms a spanning tree in $H$.

The reverse search procedure is initiated at the target $t^{*}$ and constructs a spanning tree of $H$ by reversing the local search procedure. To do this we first generate all neighbours in $H$ of any given vertex $t \in T$, in some given order. This is done by an adjacency oracle. Using the adjacency oracle at $t^{*}$ we consider neighbours of $t^{*}$ until we find a neighbour $t$ such that $L(t)=t^{*}$. We now replace $t^{*}$ by $t$ and use the adjacency oracle to find (if possible) a neighbour $s$ of $t$ such that $L(s)=t$. If such a vertex $s$ exists we move to $s$ and continue. If we reach a node $t$ for which no such neighbour $s$ exists, we backtrack by computing the parent $u=L(t)$ of $t$. We now continue from $u$ using the adjacency oracle to give the next neighbour of $u$ in order after $t$.

In order to give a formal description of a reverse search procedure it is necessary to specify:

(a) The target triangulation.

(b) The adjacency oracle.

(c) The local search procedure.

These are specified for the 3- and 2-connected cases in Sections 2 and 4, respectively.

2. 3-Connected Triangulations. In this section we describe formally how to generate all 3-connected $r$-rooted triangulations with $n$ points. To avoid trivial cases we assume $n>r \geq 3$.

2.1. Target Triangulations. For each $n>r \geq 3$ we define a target triangulation $E_{n, r}^{*}$. Figure 2.1(a) shows the target triangulation $E_{5,3}^{*}$ for $n=5$ and $r=3$. This graph is an example of a prism with apex $v_{1}$. The target triangulation $E_{7,4}^{*}$ for $n=7$ and $r=4$ is shown in Figure 2.1(b). In general, we first build a wheel on the external face $v_{1}, \ldots, v_{r}$ with centre $v_{r+1}$. Then we build a prism with apex $v_{r+1}$ in the triangle $v_{r+1} v_{r} v_{r-1}$. The edge list of $E_{n, r}^{*}$ for $n>r \geq 3$ is

$$
\begin{aligned}
& v_{1} v_{2}, v_{2} v_{3}, \ldots, v_{r-1} v_{r}, v_{1} v_{r}, \\
& v_{1} v_{r+1}, v_{2} v_{r+1}, \ldots, v_{r} v_{r+1},
\end{aligned}
$$

for the wheel, and

$$
\begin{aligned}
& v_{r-1} v_{r+2}, v_{r-1} v_{r+3}, \ldots, v_{r-1} v_{n}, \\
& v_{r} v_{r+2}, v_{r} v_{r+3}, \ldots, v_{r} v_{n}, \\
& v_{r+1} v_{r+2}, v_{r+2} v_{r+3}, \ldots, v_{n-1} v_{n},
\end{aligned}
$$

for the prism. 


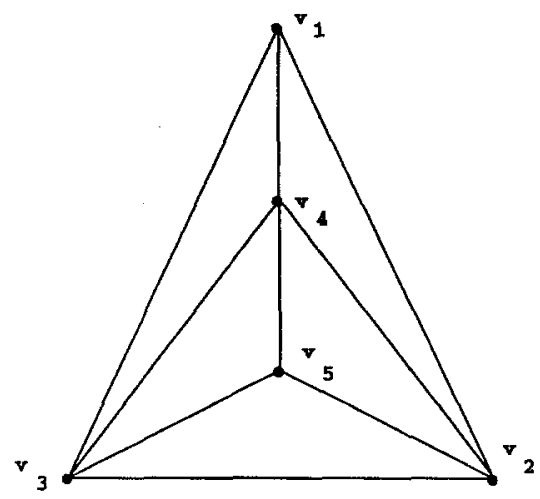

(a) $E_{5,3}^{*}$

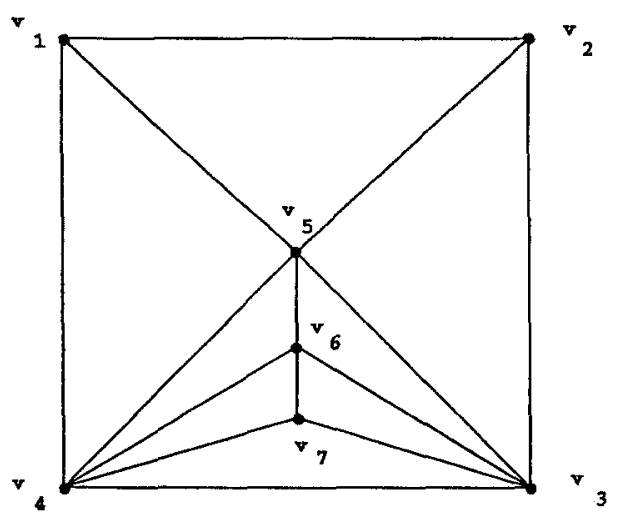

(b) $\mathbf{E}^{*} \mathbf{7 , 4}$

Fig. 2.1. Target trangulations.

2.2. Adjacency Oracle. Let $G=(V, E)$ be an $r$-rooted 3-connected triangulation and let $e=v_{a} v_{b} \in E$ be an internal edge, that is, an edge that is not on the external face. Since $e$ is internal it bounds two triangles, say $v_{a} v_{b} v_{c}$ and $v_{a} v_{b} v_{d}$. If $e^{\prime}=v_{c} v_{d}$ is not an edge in $E$ we say that $e$ is transformable and define the notation

$$
E \Delta e=E-e+e^{\prime}
$$

Note that once a transformable edge $e$ is chosen, the edge $e^{\prime}$ is well defined, and need not be specified explicitly. It is easy to see that $G^{\prime}=(V, E \Delta e)$ is also an $r$-rooted triangulation. We say that $G$ and $G^{\prime}$ are adjacent triangulations. The transformation of $E$ to $E \Delta e$ is well known, and is called a diagonal transformation. When edge $e$ is transformable, we say that we can flip edge $e . G^{\prime}$ is 3-connected if the edge $e^{\prime}$ does not join two vertices of the outer face. An edge transformation of edge $v_{1} v_{5}$ of $E_{7,4}^{*}$ (Figure 2.1(b)) produces a 2-connected triangulation, for example.

We now define an adjacency oracle that gives all adjacent triangulations to a given triangulation $G$. We assume that the edge list of $G$ is stored in some given order and let $E=\left(e_{1}, e_{2}, \ldots, e_{m}\right)$ be the ordered edge list. For $j=1, \ldots, m$ we define the adjacency oracle $\operatorname{Adj}(E, j)$ by

$$
\operatorname{Adj}(E, j)= \begin{cases}E \Delta e_{j} & \text { if } e_{j} \text { is transformable } \\ \varnothing & \text { otherwise. }\end{cases}
$$

In the ordered edge list $E \Delta e_{j}$, the edge replacing $e_{j}$ is stored in the $j$ th position of the edge list, the other edges remain in their original order. The adjacency oracle $A d j$ defines a graph $H=(T, U)$ on the set of 3-connected $r$-rooted triangulations for given $n$. The vertices $T$ of $H$ correspond to the edge sets of the triangulations. There is an edge in $U$ between the edge sets $E$ and $E^{\prime}$ if and only if

$$
E^{\prime}=\operatorname{Adj}(E, j) \quad \text { for some } j, \quad 1 \leq j \leq m .
$$

In the next section we show that $H$ is connected and give a local search procedure for $H$. 

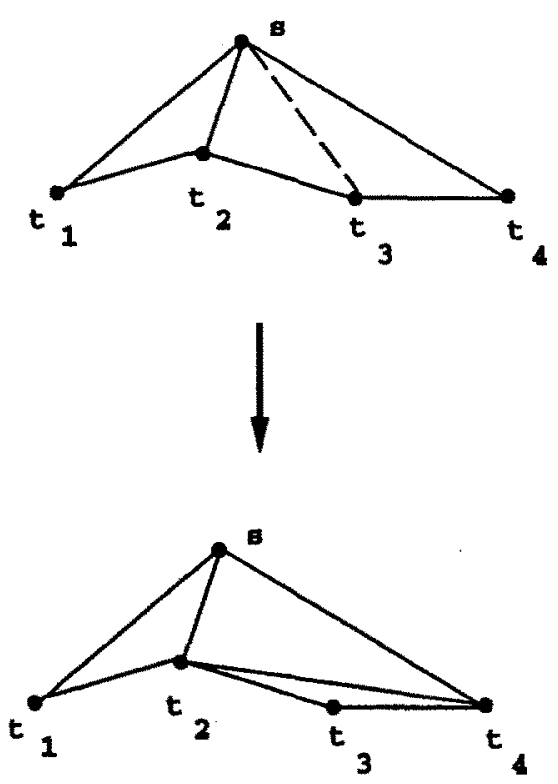

(a)

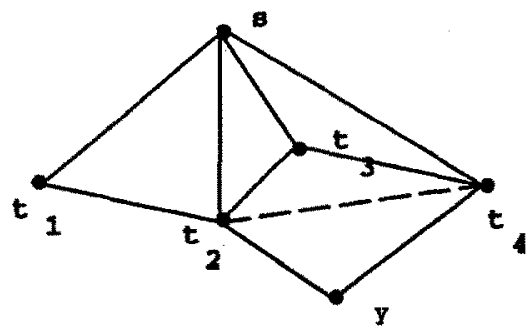

$$
1
$$

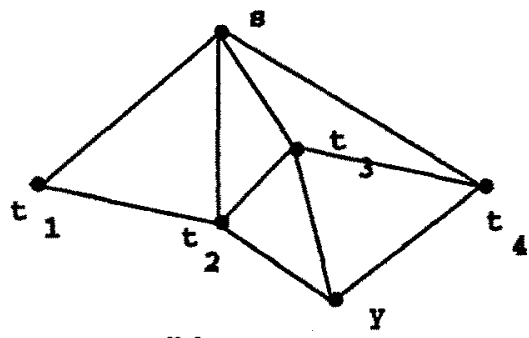

(b)

Fig. 2.2. Diagonal transformations for Lemma 2.1 .

2.3. Local Search and Wagner's Theorem. For $r=3$, the target triangulation in Section 2.1 and an implicit description of the configuration graph $H$ of Section 2.2 was given by Wagner [13] and also appears in the book by Ore [9]. Wagner proved that $H$ is connected when $r=3$. A somewhat simpler proof is contained in [9] and this proof is suitable for defining an efficient local search procedure. We give a proof based on a similar idea for the general case $r \geq 3$. We begin with a basic lemma which is illustrated in Figures 2.2(a) and (b). We say that a vertex $v$ has consecutive neighbours $t_{1}, t_{2}, \ldots, t_{k}$ if these neighbours occur in consecutive counterclockwise order in the unique planar embedding of the $r$-rooted triangulation.

LEMMA 2.1. Let $s$ be a vertex in an $r$-rooted triangulation $G$ with four consecutive neighbours $t_{1}, t_{2}, t_{3}, t_{4}$, where $t_{1}$ is on the external face and $t_{2}$ is not. Then either $s t_{3}$ is transformable or $t_{2} t_{4}$ is an edge of $G$ which is transformable. The edge transformation preserves 3-connectivity.

PROOF. Since $t_{1}, t_{2}, t_{3}, t_{4}$ are consecutive neighbours of $s$, the edge $s t_{3}$. bounds the triangles $s t_{2} t_{3}$ and $s t_{3} t_{4}$. If $t_{2} t_{4}$ is not an edge, then $s t_{3}$ is transformable. Otherwise $t_{2} t_{4}$ is an internal edge and $t_{3}$ lies inside the triangle $s t_{2} t_{4}$. The edge $t_{2} t_{4}$ bounds two triangles $x t_{2} t_{4}$ and $y t_{2} t_{4}$ of the triangulation. One of these triangles, say $x t_{2} t_{4}$, lies inside $s t_{2} t_{4}$ 
and the other outside ( $x$ may or may not be the same as $t_{3}$ ). Therefore $x$ and $y$ are not adjacent so $t_{2} t_{4}$ is transformable.

Suppose $G$ is 3-connected. It suffices to show that the new edge contains an internal vertex. The edge transformation produces either the new edge $t_{2} t_{4}$ or the new edge $t_{3} x$. In the first case $t_{2}$ is internal by hypothesis and in the second case $t_{3}$ is internal since it lies inside triangle $s t_{2} t_{4}$. In both cases 3 -connectivity is preserved.

Before formally defining the local search algorithm, we illustrate it on the example $G_{1}$ in Figure 2.3. The idea is to compare vertices $v_{1}, v_{2}, v_{3}, \ldots$ in $G_{1}$ with the corresponding vertices in the target, $E_{7,4}^{*}$, shown in Figure 2.1(b). When the first vertex is found that differs from the target, the lemma is applied. This is repeated until the target is reached. Comparing the two triangulations $G_{1}$ and $E_{7.4}^{*}$ we see that $d\left(v_{1}\right)=3$ in $G_{1}$, matching its counterpart in the target, but $d\left(v_{2}\right)=4$ does not. We apply the lemma with $s=v_{2}$, $t_{1}=v_{1}, t_{2}=a, t_{3}=b$, and $t_{4}=v_{3}$. Since $a v_{3}$ is an edge, $v_{2} b$ is not transformable so we flip $a v_{3}$ getting $G_{2}$. In $G_{2}, v_{1}$ again has the correct degree and $v_{2}$ has degree 4 . Applying the lemma again it is now possible to flip $v_{2} b$ reducing the degree of $v_{2}$ to 3
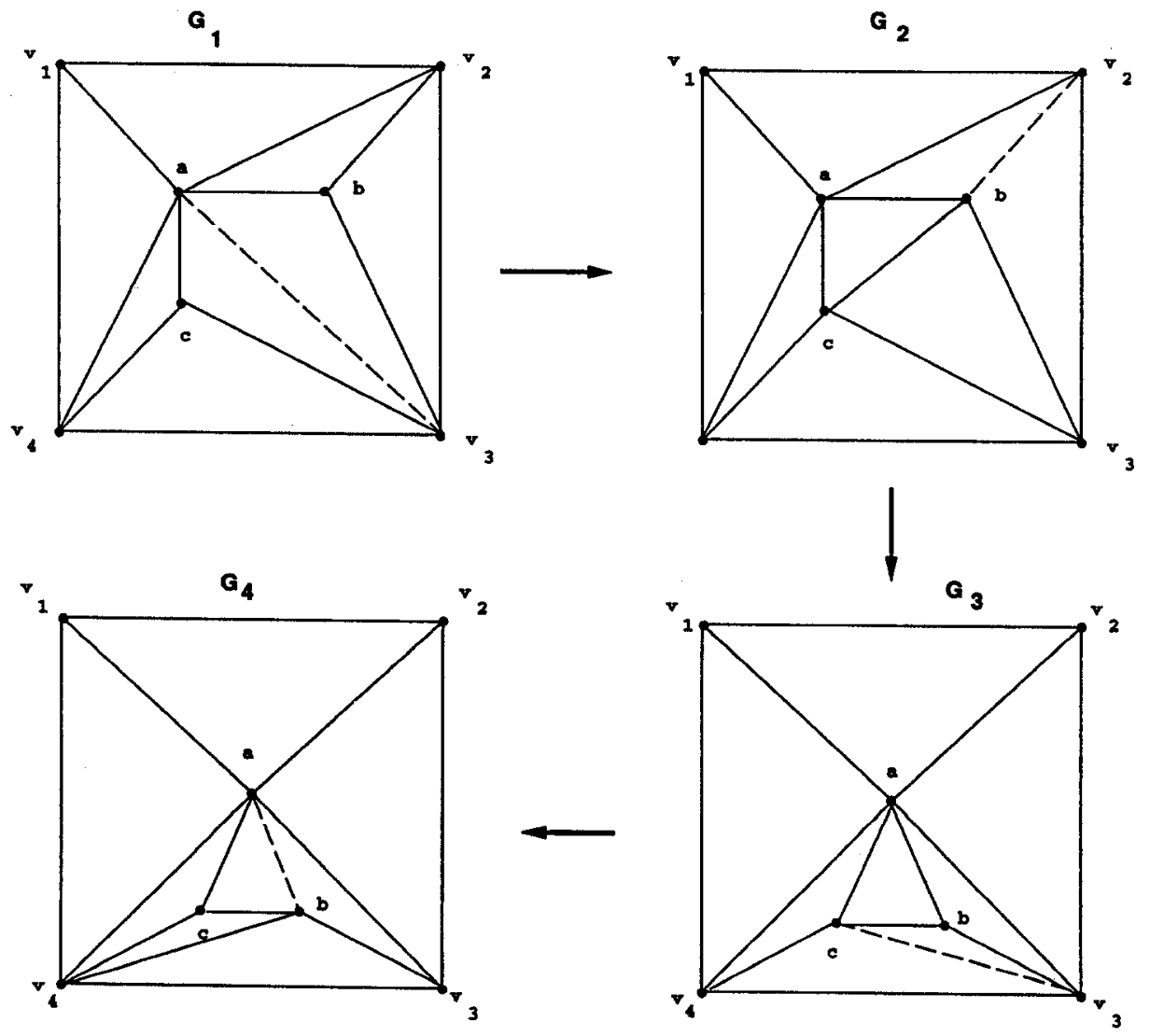

Fig. 2.3. Illustrating LocalSearch (selected edge shown dashed). 
procedure LocalSearch $(E, n, r)$;

$I^{*}$ return a transformable edge or null if $E=E_{n, r}^{*} *$

$/ *$ create a wheel with centre $v_{r+1} * /$

$i:=1$;

while $d\left(v_{i}\right) \doteq 3$ and $i \leq r-2$

$$
i:=i+1 \text {; }
$$

\section{endwhile}

if $i \leq r-2$ then $\quad / * d\left(v_{i}\right)>3 / *$

let $t_{1}:=v_{i-1}, t_{2}, t_{3}, t_{4}$ be consecutive neighbours of $v_{i}$ in counterclockwise order;

if $t_{2} t_{4}$ is not an edge then return $\left(s t_{3}\right)$

endif

else return $\left(t_{2} t_{4}\right)$

$1^{*}$ Transform the triangle $v_{r+1} v_{r-1} v_{r}$ to a prism $* /$

let $s:=$ the vertex adjacent to $v_{1}, \ldots v_{r}$;

lasts $:=v_{1}$;

while $s$ is adjacent to exactly one internal vertex $a$ that is not lasts

endwhile

$$
\text { lasts }:=s ; \quad s:=a ;
$$

if $d(s)=3$ return $(\varnothing) ; \quad /^{*} E=E_{n, r}^{*} *$ /

$l^{*}$ We have found the first vertex where $E$ is different from the target*/

let $t_{1}:=v_{r}, t_{2}, t_{3}, t_{4}$ be consecutive neighbours of $s$ in counterclockwise order;

if $t_{2} t_{4}$ is not an edge then return $\left(s t_{3}\right)$

else return $\left(t_{2} t_{4}\right)$;

Fig. 2.4. Local search procedure for 3-connected triangulations.

and obtaining $G_{3}$. In $G_{3}$ the degrees of both $v_{1}$ and $v_{2}$ are both 3 so we have a wheel with centre $a$, external face $v_{1}, \ldots, v_{4}$, and all remaining vertices in the triangle $a v_{3} v_{4}$. We now transform this triangle into the target prism shown in Figure 2.1(a). For this purpose imagine vertices $v_{1}$ and $v_{2}$ along with incident edges have been deleted.

Inside triangle $a v_{3} v_{4}$ vertex $a$ has degree 4 so we apply the lemma with $t_{1}=v_{4}, t_{2}=c$, $t_{3}=b$, and $t_{4}=v_{3}$, getting the transformable edge $c v_{3}$. The resulting triangulation $G_{4}$ has similar structure and the lemma is applied again to the same vertex set giving the transformable edge $a b$. This diagonal transformation leads to the target $E_{7,4}^{*}$, with $v_{5}=a, v_{6}=c$, and $v_{7}=b$.

The above method is stated formally as procedure LocalSearch in Figure 2.4. Let $E$ be the edge list of a 3-connected $r$-rooted triangulation on $n$ points that is not the target triangulation. LocalSearch $(E, n, r)$ returns a transformable edge $e \in E$. The next theorem, which generalizes a theorem of Wagner [13], shows that repeated application of this procedure leads to the target triangulation.

THEOREM 2.1 (Generalized Wagner's Theorem).

(a) If $E$ is a 3-connected triangulation, LocalSearch $(E, n, r)$ returns a transformable edge $e \in E$ which preserves 3-connectivity.

(b) By repeated application of LocalSearch to the transformed edge set $E \Delta \theta$ the target triangulation $E_{n, r}^{*}$ is reached. 
PROOF. For part (a) of the theorem, we apply Lemma 2.1. If $e$ is chosen in the first part of the procedure, $s$ and $t_{1}$ are on the external face and $d(s) \geq 4$. By 3-connectivity, $t_{2}$ is an internal vertex. If $e$ is chosen in the second part of the procedure, $t_{2}$ is inside the triangle $s v_{r-1} v_{r}$ and so it is internal. In both cases the lemma applies, the edge chosen is transformable and 3-connectivity is preserved.

For part (b), we first show that $E$ is transformed to a triangulation with $d\left(v_{i}\right)=3$ for $i=1, \ldots, r-2$. Indeed, suppose $i$ is the smallest index in this range such that $d\left(v_{i}\right)>3$. Vertex $v_{i}$ is selected in this part of the procedure. Since $v_{i}$ is on the external face and the triangulation is 3-connected, the second consecutive neighbour of $v_{i}$ must be internal and so Lemma 2.1 can be applied. Suppose $e$ is the transformable edge provided by the lemma and consider the triangulation $E^{\prime}=E \Delta e$. Either $d\left(v_{i}\right)$ has been decreased by one, or LocalSearch $\left(E^{\prime}, n, r\right)$ returns an edge $e^{\prime}$ such that the triangulation $E^{\prime} \Delta e^{\prime}$ has this property.

We must show that the diagonal transformation does not increase the degrees of $v_{1}, \ldots, v_{i-1}$. There are two cases depending on the choice of edge in the lemma. First, if $s t_{3}$ is transformable, the new edge is $t_{2} t_{4}$. Since $s=v_{i}$ and $t_{1}=v_{i-1}$ are external, 3connectivity implies that $t_{4}$ is either internal or $t_{4}=v_{i+1}$. In either case the transformation does not increase the degrees of $v_{1}, \ldots, v_{i-1}$. In the second case, $t_{2} t_{4}$ is an edge, and the new edge is $x t_{3}$. Suppose $x=v_{k}$ for some $1 \leq k \leq i-1$. Now $t_{4}$ must also be external, for otherwise $v_{k}$ is adjacent to $v_{k-1}, v_{k+1}, t_{2}$, and $t_{4}$ before the transformation, violating the condition that $d\left(v_{k}\right)=3$. However, if $t_{4}$ is external, we have $t_{4}=v_{i+1}$ by 3 -connectivity. Since $t_{4}$ is also adjacent to $x=v_{k}$, we must have $t_{4}=v_{r}$ so $i=r-1$, contradicting the choice of $i$ by LocalSearch. Therefore $x$ is either internal or external with index greater than $i$ and the degrees of $v_{1}, \ldots, v_{i-1}$ are preserved. It follows that repeated application of LocalSearch produces a triangulation with $d\left(v_{i}\right)=3$ for $i=1, \ldots, r-2$.

We now have a triangulation that is a wheel with external face $v_{1}, \ldots, v_{r}$, with centre, say, $s$ and with the remaining vertices in the triangle $s v_{r-1} v_{r}$. We label $s$ as $v_{r+1}$. The triangulation inside this triangle is to be transformed into a prism. Firstly, if $s$ is adjacent to more than one internal vertex, we apply Lemma 2.1 to the four consecutive neighbours of $s$ in counterclockwise order starting from $v_{r}$. Note that since $v_{r-1} v_{r}$ is an edge of the external face, the second and third consecutive neighbours of $s$ are internal. If we do a diagonal transformation on the edge returned, either the degree of $s$ is reduced by one, or this happens on the following application of LocalSearch and subsequent diagonal transformation. We continue in this way, until $s$ is adjacent to one internal vertex, say, $a$. We label $a$ as $v_{r+2}$. It follows that $a$ is adjacent to both $v_{r-1}$ and $v_{r}$. Setting lasts $=s$ and $s=a$ we now repeat essentially the same steps until the new vertex $s$ has exactly one internal vertex (say $a=v_{r+3}$ ) that is different from lasts. We move down the chain $v_{r+1}, v_{r+2}, \ldots$ until a vertex is found that differs from the corresponding vertex in the target, and apply the above procedure.

It can be verified that once the vertices $v_{r+1}, v_{r+2}, \ldots$ have degrees corresponding to the target they are never involved in further edge transformations. Therefore the procedure is finite and the theorem is proved.

3. Implementation of Reverse Search Procedure. In this section we give the details on how the reverse search technique is used to generate triangulations. We begin by 


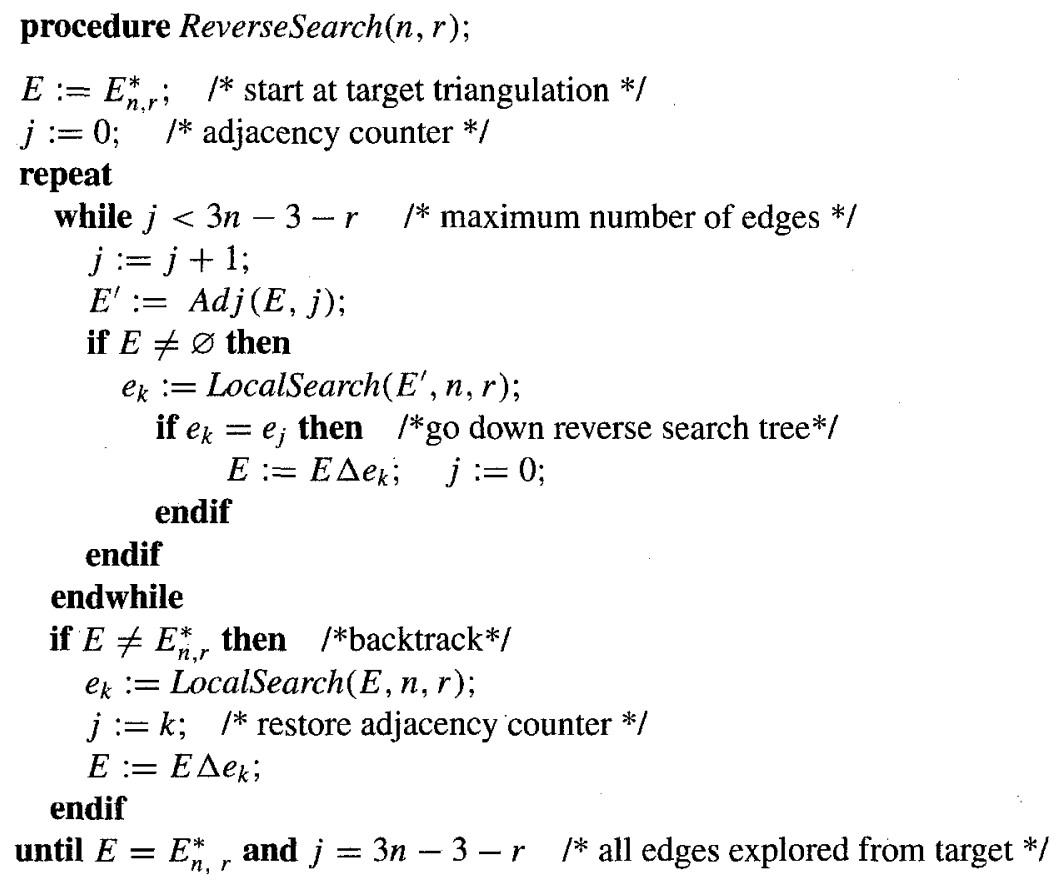

Fig. 3.1. Pseudocode for ReverseSearch.

giving the procedure ReverseSearch which is the standard reverse search procedure, essentially as given in [2]. The reader unfamiliar with reverse search is referred to this paper for further description, formal proofs of correctness, and complexity analysis. However, reference to the informal description in Section 1 and the code ReverseSearch shown in Figure 3.1 should give the essential idea.

We now give a simple data structure that allows LocalSearch and $A d j$ to be implemented in $O(n)$ time. After a diagonal transformation, the data structure can be updated in $O(n)$ time. This leads to an implementation of ReverseSearch in $O\left(n^{2} g(n, r)\right)$ time and $O(n)$ space. The data structure used is the doubly connected edge list, or DCEL, of Muller and Preparata [10], a description of which can be found in [11]. Each edge $a b$ of the triangulation is represented by a corresponding edge node in the DCEL. The edge node for $a b$ contains the vertex labels $a$ and $b$ and two pointers:

- A pointer to the edge node corresponding to the edge $a c$ which is next in counterclockwise order about $a$ after $a b$.

- A pointer to the edge node corresponding to the edge $b d$ which is next in counterclockwise order about $b$ after $a b$.

The edge nodes are stored in an array. Each entry of the array contains two data fields for the vertex labels and two pointer fields containing the array indices for the two edge nodes described above. The data structure for $E_{5,3}^{*}$ is shown in Table 3.1. This data structure has the following properties: 
Table 3.1. DCEL data structure for $E_{5,3}^{*}$.

\begin{tabular}{ccccc}
\hline Index & Vertex 1 & Vertex 2 & $\begin{array}{c}\text { Next } \\
\text { vertex 1 }\end{array}$ & $\begin{array}{c}\text { Next } \\
\text { vertex 2 }\end{array}$ \\
\hline 1 & $v_{1}$ & $v_{2}$ & 2 & 5 \\
2 & $v_{1}$ & $v_{3}$ & 3 & 4 \\
3 & $v_{1}$ & $v_{4}$ & 1 & 7 \\
4 & $v_{2}$ & $v_{3}$ & 1 & 8 \\
5 & $v_{2}$ & $v_{4}$ & 6 & 3 \\
6 & $v_{2}$ & $v_{5}$ & 4 & 9 \\
7 & $v_{3}$ & $v_{4}$ & 2 & 9 \\
8 & $v_{3}$ & $v_{5}$ & 7 & 6 \\
9 & $v_{4}$ & $v_{5}$ & 5 & 8 \\
\hline
\end{tabular}

(P1) From an edge node $a b$, the edge nodes of all of the edges of the triangles $a b c$ and $a b d$ can be obtained in constant time.

(P2) From an edge node $a b$, the edges adjacent to $a$ (or $b$ ) may be obtained in counterclockwise order, starting from $a b$, at a cost of constant time per edge.

Consider first the implementation of the adjacency oracle $\operatorname{Adj}(E, j)$. The edge counter $j$ is used as an index into the array storing the edge nodes. For given $j$, an edge $a b$ is located in constant time. By (P1), the two triangles $a b c$ and $a b d$ bounded by $a b$ can also be determined in constant time. A sequential search of the edge nodes is used to see if $c d$ is an edge. If so, the empty set is returned. If not, a diagonal transformation is performed on edge $a b$. This can be done in constant time. The updates to DCEL are shown in Table 3.2. It is assumed that $d$ is the next neighbour of $a$ in counterclockwise or-

Table 3.2. DCEL before and after diagonal transformation on $a b$.

\begin{tabular}{ccccc}
\hline Index & Vertex 1 & Vertex 2 & $\begin{array}{c}\text { Next } \\
\text { vertex 1 }\end{array}$ & $\begin{array}{c}\text { Next } \\
\text { vertex 2 }\end{array}$ \\
\hline (a) Before \\
r1 & $a$ & $b$ & $\mathrm{r} 3$ & $\mathrm{r} 4$ \\
$\mathrm{r} 2$ & $a$ & $c$ & $\mathrm{r} 1$ & \\
$\mathrm{r} 3$ & $a$ & $d$ & & $\mathrm{r} 5$ \\
$\mathrm{r} 5$ & $b$ & $c$ & & $\mathrm{r} 2$ \\
\hline
\end{tabular}

(b) After

\begin{tabular}{lllll}
\hline $\mathrm{r} 1$ & $c$ & $d$ & $\mathrm{r} 2$ & $\mathrm{r} 5$ \\
$\mathrm{r} 2$ & $a$ & $c$ & $\mathrm{r} 3$ & \\
$\mathrm{r} 3$ & $a$ & $d$ & & $\mathrm{r} 1$ \\
$\mathrm{r} 4$ & $b$ & $c$ & & $\mathrm{r} 1$ \\
$\mathrm{r} 5$ & $b$ & $d$ & $\mathrm{r} 4$ & \\
\hline
\end{tabular}


der after $b$, and fields not shown are unchanged. Observe that if edge $a b$ is transformable and its location in DCEL is known, then the transformation takes $O(1)$ time. Testing transformability takes $O(n)$ time since the edges are not stored in order in DCEL.

Next consider the implementation of LocalSearch. This procedure finds the first vertex with degree greater than that of the target, where the vertices are checked in order around the outer face and then from the centre of the prism to the external face. By property (P2), this can be done in time proportional to the number of edges, and the traversal of the vertices in the given order can easily be achieved with the DCEL structure. Once the first vertex of the triangulation differing from the target is found, this becomes vertex $s$ of Lemma 2.1. A scan around $s$ produces the vertices $t_{1}, t_{2}, t_{3}, t_{4}$. A linear scan of the edge nodes determines which of the cases of the lemma apply. The total time required by LocalSearch is therefore $O(n)$.

We can now analyse ReverseSearch. The while loop of the repeat block dominates the computation. For each triangulation and each edge in the triangulation it is necessary to call $A d j$ and possibly LocalSearch. The total time for this step is therefore $O\left(n^{2} g(n, r)\right)$.

4. 2-Connected Triangulations. In this section we describe the modifications needed to produce all 2-connected triangulations. To avoid cases covered already we may assume $n \geq r \geq 4$. The modifications required are new target triangulations and a slightly different local search procedure. The adjacency oracle described in Section 2.2 is valid without change.

Figure 4.1 shows the target triangulation $F_{6,5}^{*}$. In general the target $F_{n, r}^{*}$ is specified by:

(a) Creating a star-shaped triangulation of the outer face from vertex $v_{r}$.

(b) Creating a prism with apex $v_{r-2}$ in the triangle $v_{r-2} v_{r-1} v_{r}$ with the remaining $n-r$ points, if any.

The edge list of $F_{n}, r^{*}$ for $n \geq r \geq 4$ is

$$
\begin{aligned}
& v_{1} v_{2}, v_{2} v_{3}, \ldots, v_{r-2} v_{r-1}, \\
& v_{1} v_{r}, v_{2} v_{r}, \ldots, v_{r-1} v_{r},
\end{aligned}
$$

for the outer face, and, when $n>r$,

$$
\begin{aligned}
& v_{r-2} v_{r+1}, v_{r+1} v_{r+2}, v_{r+2} v_{r+3}, \ldots, v_{n-1} v_{n} \\
& v_{r-1} v_{r+1}, v_{r-1} v_{r+2}, \ldots, v_{r-1} v_{n} \\
& v_{r} v_{r+1}, v_{r} v_{r+2}, \ldots, v_{r} v_{n}
\end{aligned}
$$

for the prism.

The local search procedure for generating 2-connected triangulations is based on the same idea as described in Section 2.3 for 3 -connected triangulations. Let $E$ be the edge list of a triangulation that is not the target $F_{n, r}^{*}$. We check the vertices sequentially about the external face $v_{1}, v_{2}, \ldots, v_{r-3}$ reducing the degree of vertex $v_{1}$ to 2 , and the other degrees to 3 . When this has been accomplished, the outer face has been triangulated as a star from vertex $v_{r}$ and all internal vertices lie inside the triangle $v_{r-2} v_{r-1} v_{r}$. We now 

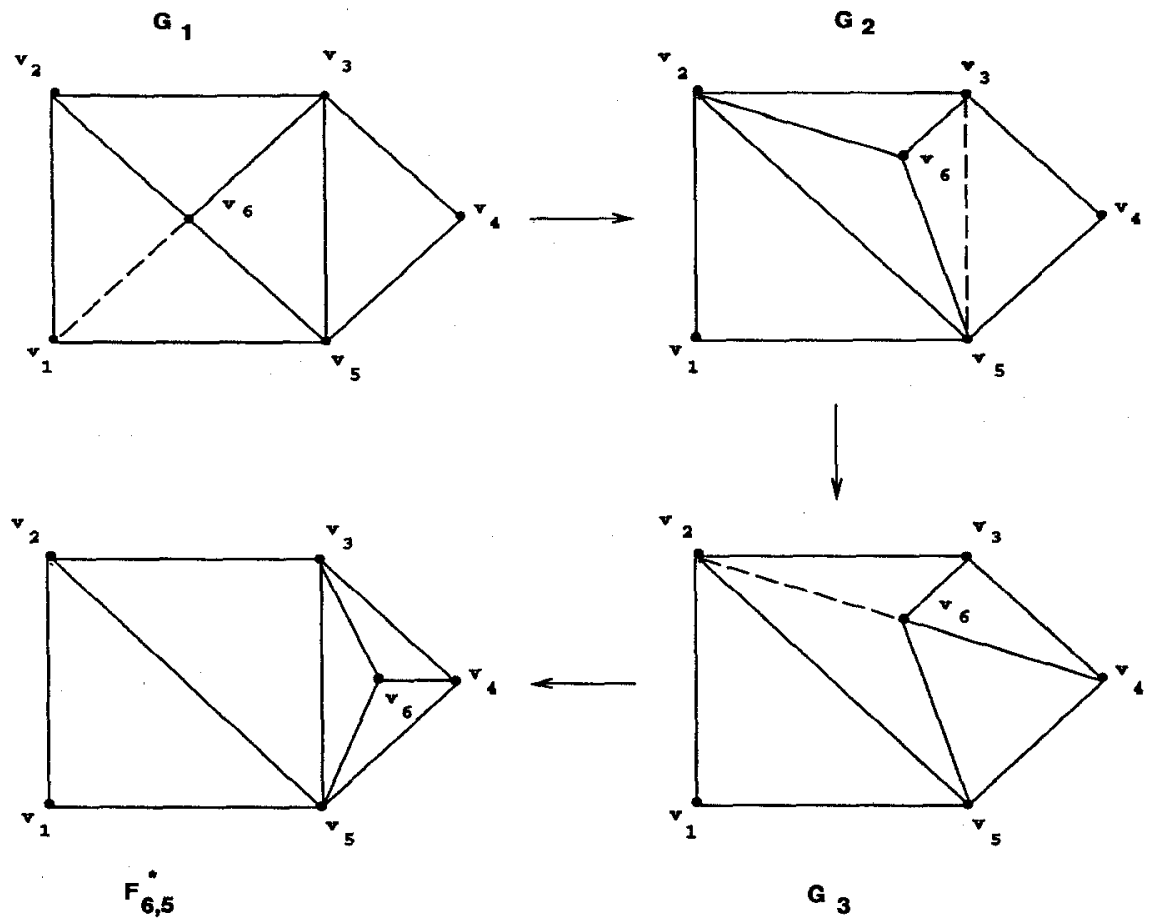

Fig. 4.1. Illustrating target and LocalSearch2 (selected edge shown dashed).

transform this triangle into a prism as described in Section 2.3. The transformation of the outer face to the target is based on the following lemma.

LEMMA 4.1. For $i=1,2, \ldots, r-3$, if $v_{i}$ is adjacent to consecutive vertices $v_{r}, t_{1}, t_{2}$, then either $v_{i} t_{1}, v_{i} t_{2}$, or $v_{r} t_{2}$ is a transformable edge.

PROOF. If $v_{i} t_{1}$ is not transformable, $v_{r} t_{2}$ must be an edge, and $t_{1}$ lies inside the triangle $v_{i} v_{r} t_{2}$. First suppose $t_{2} \neq v_{i+1}$ (Figure 4.2(a)). Then $v_{i}$ has at least one more consecutive neighbour after $t_{2}$. Therefore $v_{i} t_{2}$ is an internal edge bounding triangles $v_{i} t_{1} t_{2}$ and $v_{i} t_{2} a$ for some vertex $a$. Since $a$ cannot be adjacent to $t_{1}$, edge $v_{i} t_{2}$ is transformable. Otherwise $t_{2}=v_{i+1}$ and since $i \leq r-3, v_{i+1} v_{r}$ is an internal edge bounding triangles $x v_{i+1} v_{r}$ and $y v_{i+1} v_{r}$, for some vertices $x$ and $y$ (Figure 4.2(b)). One of these triangles, say $x v_{i+1} v_{r}$, must lie inside $v_{i} v_{r} v_{i+1}$ and the other outside. Note that $x$ may or may not be identical to vertex $t_{1}$. Vertices $x$ and $y$ cannot be adjacent, so $v_{r} t_{2}$ is transformable.

Based on Lemmas 2.1 and 4.1 we present in Figure 4.3 the procedure LocalSearch2 which transforms and 2-connected rooted triangulation to the target. An application of the procedure is shown in Figure 4.1. Analagous to Theorem 2.1 we have the following result. 


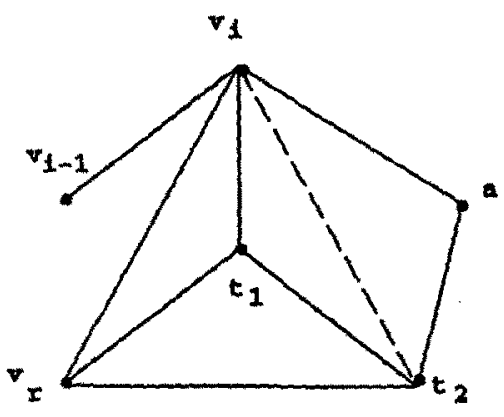

(a)

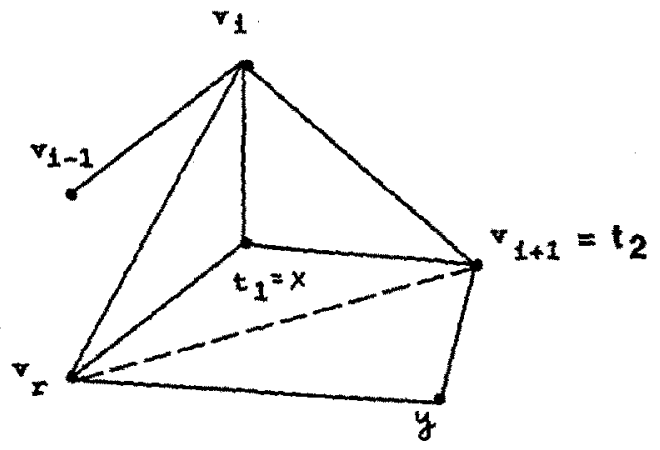

(b)

Fig. 4.2. Ilustrating Lemma 4.

\section{THEOREM 4.1.}

(a) If $E$ is a 2-connected triangulation, LocalSearch2( $E, n, r)$ returns a transformable edge e.

(b) By repeated application of LocalSearch 2 to the transformed edge set $E \Delta$ e the target triangulation $F_{t, r}^{*}$ is reached.

To prove the theorem, Lemma 4.1 is applied when the edge is selected in the first step

procedure LocalSearch $2(E, n, r)$;

* Assume $n \geq r \geq 4 * /$

$l^{*}$ Create a star triangulation from $v_{r} * /$

if the degree of $v_{1}>2$ or the degree of $v_{i}>4$ for some $i=2,3, \ldots, r-3$ then

choose the smallest such $i$ and suppose $v_{i}$ is adjacent to consecutive vertices $v_{r}, t_{1}, t_{2}$;

if $v_{r} t_{2}$ is not an edge then return $\left(v_{i} t_{1}\right)$;

if $t_{2} \neq v_{i+1}$ then return $\left(v_{i} t_{2}\right)$

endif else return $\left(v_{r} t_{2}\right)$;

$l^{*}$ Transform the triangle $v_{r-2} v_{r-1} v_{r}$ to a prism $*$

$s=v_{r-2}$;

if $s$ is adjacent to exactly one internal vertex $a$ then

lasts $=s ; \quad s=a ;$

while $s$ is adjacent to exactly two internal vertices lasts and $a$

lasts $=s ; \quad s=a$

endwhile

endif

$1 *$ We have found the first vertex where $E$ is different from the target $*$ ]

Suppose $t_{1}=v_{r}, t_{2}, t_{3}, t_{4}$ are consecutive vertices adjacent to $s$ in counterclockwise order;

if $t_{2} t_{4}$ is not an edge then return(st 3 )

else return $\left(t_{2} t_{4}\right)$

Fig. 4.3. Local search for 2-connected triangulations. 
of LocalSearch2. When the edge is selected in the second part, Lemma 2.1 is applied. The proof is similar to Theorem 2.1 and the details are omitted.

Using LocalSearch2 and $F_{n, r}^{*}$ in place of LocalSearch and $E_{n, r}^{*}$, respectively, in ReverseSearch we obtain an algorithm for generating all 2-connected triangulations. This algorithm can be implemented using the DCEL data structure described in Section 3. Using an analysis similar to that given in Section 3, we can show that LocalSearch 2 can also be implemented in $O(n)$ time. We conclude that the modified ReverseSearch procedure produces all 2-connected triangulations in $O\left(n^{2} f(n, r)\right)$ time and $O(n)$ space.

5. Concluding Remarks. We have presented efficient algorithms to generate all 2and 3-connected rooted triangulations. The algorithm for generating 3-connected triangulations was programmed using essentially the method described here with a slightly different data structure. The program is available by FTP to mutt.cs.mcgill.ca. Please use the login name ftp, give your full email address as password and change to directory pub/tri. The program is written in $C$ and called 3tri.c. It was tested for all $n$ and $r$ in the range $3 \leq r<n \leq 12$, and the number of triangulations matched the Tutte formula for $g(n, r)$. The largest problem solved, with $n=13, r=3$, and $g(13,3)=6,369,883$, took 270 minutes on a Silicon Graphics workstation.

The reverse search method used to generate all $r$-rooted triangulations is the so-called naive method. The dominant step in the algorithm is going down the reverse search tree, which is essentially testing whether

$$
e_{j}=\operatorname{LocalSearch}\left(E \Delta e_{j}, n, r\right)
$$

for each edge $e_{j}$ in triangulation $E$. In the naive method, if $e_{j}$ is transformable, the transformation is made and then LocalSearch is applied, in time $O(n)$, to determine if $e_{j}$ is the chosen edge. In many applications of reverse search, this test can be done more efficiently [2]. In our case, many candidate edges $e_{j}$ can be rejected without computing $E \Delta e_{j}$ and calling LocalSearch. It is an open problem whether the test (5.1) can be done in $O$ (1) time. The constants implied by the big-O notation are important, since this problem is feasible only for small values of $n$.

Finally we mention how the results in this paper can be used to compute all nonisomorphic triangulations of the sphere. A list of these triangulations can be obtained from a list of all 3-rooted triangulations by removing isomorphisms. For each triangulation of the sphere on $n \geq 4$ points there are $2 n-4$ triangles. Each can become the outer face of a planar triangulation and can be labelled in six ways. Some of these $12 n-24$ rooted triangulations may be isomorphic, but if the original triangulation of the sphere has no symmetries (which is possible), all will be distinct. Therefore each triangulation of the sphere may appear up to $12 n-24$ times as a rooted triangulation, and duplicates would have to be removed by isomorphism testing. The time to do this can be greatly reduced by various filtering techniques. For example, a rooted triangulation can be rejected if the degree sequence of the outer triangle is not lexicographically maximal over the degree sequences of all interior triangles. The remaining triangulations are tested for isomorphism. 
An important open question is whether the reverse search method can be applied to generate triangulations of the sphere directly without the need for isomorphism testing. More generally it would be of interest to generate all 3-connected planar graphs, or dually, all distinct combinatorial types of three-dimensional polyhedra without repetitions.

Acknowledgements. The author is grateful to Antoine Deza, Komei Fukuda, Bill Lenhart, S. Negami, G. Toussaint, the students of his graduate class in Combinatorial Algorithms at McGill and an anonymous referee for discussions and comments on the results in this paper. The author thanks Masakazu Kojima for providing facilities during the spring of 1993 in his laboratory at the Tokyo Institute of Technology, where the programming was done.

Note Added in Proof. Gunnar Brinkman and Brendan McKay (private communication) have recently found an efficient method for generating planar unrooted triangulations without isomorphism testing, solving the above open problem.

\section{References}

[1] D. Avis and K. Fukuda, A Pivoting Algorithm for Convex Hulls and Vertex Enumeration of Arrangements and Polyhedra, Distrete Comput. Geom., 8 (1992), 295-313.

[2] D. Avis and K. Fukuda, Reverse Search for Enumeration, Discrete Appl. Math., 6 (1996), 21-46.

[3] P. Bose, T. Shermer, G. T. Toussaint, and B. Zhu, Guarding Polyhedral Terrains, Technical Report SOCS 92.20, McGill University, 1992. Comput. Geom. Theory Appl. (to appear).

[4] R. Bowen and S. Fisk, Generation of Triangulations of the Sphere, Math. Comp., 21 (1967), $250-252$.

[5] W. G. Brown, Enumeration of Triangulations of the Disk, Proc. London Math. Soc, 14 (1964), 746-768.

[6] A. Deza, K. Fukuda, and V. Rosta, Wagner's Theorem and Combinatorial Enumeration of 3-Polytopes, in RIMS Kokyuroku 872, ed. H. Imai, pp. 30-34, Kyoto University, 1994.

[7] M. B. Dillencourt, Polyhedra of Small Order and Their Hamiltonian Properties, Technical Report 92-91, Information and Computer Science, University of California, Irvine, September 1992.

[8] F. Hurtado, Private communication.

[9] O. Ore, The Four-Color Problem. Academic Press, New York, 1967.

[10] F. P. Preparata and D. E. Muller, Finding the Intersection of Two Convex Polyhedra, Theoret. Comput. Sci., 7, (1978), 217-236.

[11] F. P. Preparata and M. I. Shamos, Computational Geometry, Springer-Verlag, New York, 1985.

[12] W. T. Tutte, A Census of Planar Triangulations, Canad. J. Math., 14 (1962), 21-38.

[13] K. Wagner, Bemerkungen zum Vierfarbenproblem, Jahresber. Deutsch. Math. -Verein., 46(1) (1936), $26-32$. 